\title{
TRANSTORNOS DE ESTRESSE AGUdO E PÓS-TRAUMÁTICO*
}

\section{Paula Maria Serafim ${ }^{1}$, Marcelo Feijó de Mello ${ }^{2}$}

Os transtornos de estresse agudo e de estresse pós-traumático são duas condições específicas causadas pela violência. São quadros graves, com repercussões biológicas, psicológicas e sociais profundas. Foi criado programa para atender e estudar a violência (Prove), em 2004. A partir de maio de 2009, houve mudança do atendimento que passou a ser multidisciplinar. Foram realizados 1.445 atendimentos dessa data até novembro de 2009 e incluídos 76 novos pacientes adultos e 27 crianças. Os eventos traumáticos mais frequentes foram assalto e sequestro (adultos) e assassinato de um familiar e abuso sexual (crianças). O sistema multidisciplinar é efetivo e apresenta alta adesão (87\%/6 meses).

Descritores: Transtornos de Estresse Pós-Traumáticos; Violência; Saúde Mental; Diagnóstico; Terapêutica.

\section{ACute AND Post-Traumatic Stress Disorder ${ }^{\dagger}$}

Acute and post-traumatic stress disorders are two conditions caused specifically by violence. These are severe disorders, with deep biological, psychological and social effects. The Program to assist in and study violence (PROVE) was created in 2004. As of May 2009 the assistance changed to a multidisciplinary approach. A total 1,445 cases were seen between that date and November 2009, and new patients were included, 76 adults and 27 children. The most common traumatic events were hold-ups and kidnapping (adults) and murder of a relative and sexual abuse (children). The multidisciplinary system is effective with high adherence (87\%/6 months).

Descriptors: Stress Disorders, Post-Traumatic; Violence; Mental Health; Diagnosis; Therapeutics.

\section{TRANSTORNOS DE ESTRÉS AGUDO Y POSTRAUMÁTICO}

Los transtornos de estrés agudo y de estrés postraumático son dos condiciones específicas causadas por la violencia. Son cuadros graves, con repercusiones biológicas, psicológicas y sociales profundas. Se creó un programa para atender y estudiar la violencia (PROVE) en 2004. A partir de mayo de 2009 hubieron cambios en la atención, que pasó a ser multidisciplinaria. Se realizaron 1.445 atenciones desde tal fecha hasta noviembre de $2009 \mathrm{y}$ se incluyeron 76 nuevos pacientes adultos y 27 niños. Los eventos traumáticos más frecuentes fueron asalto y secuestro (adultos) y asesinato de un familiar y abuso sexual (niños). El sistema multidisciplinario es efectivo y de alta adhesión (87\%/6 meses).

Descriptores: Transtornos por Estrés Postraumático; Violencia; Salud Mental; Diagnóstico; Terapéutica.

\footnotetext{
*Trabalho apresentado no XI Encontro de Pesquisadores em Saúde Mental e Enfermagem Psiquiátrica. Ribeirão Preto, SP, Brasil, 2010.

${ }^{\dagger}$ This study was presented in the 11st Meeting of Researchers in Mental Health and Psychiatric Nursing. Ribeirão Preto, SP, Brazil, 2010.
}

1-Enfermeira, Especialista em Saúde Mental, Programa de Atendimento e Pesquisa em Violência, Departamento de Psiquiatria, Universidade Federal de São Paulo, SP, Brasil. E-mail: paulinhaserafim@yahoo.com.br 2-Médico Psiquiatra, Doutor em Psiquiatria, Professor Afiliado, Departamento de Psiquiatria, Universidade Federal de São Paulo, SP, Brasil. E-mail: $\underline{\text { mf-mello@uol.com.br }}$

Autor Correspondente: Marcelo Feijó Mello Endereço para Correspondência: Universidade Federal de São Paulo, Departamento de Psiquiatria. Rua Dr. Barcelar, 334, Bairro Ibirapuera, CEP 04026-001, São Paulo, SP, Brasil. E-mail: mf-mello@uol.com.br 


\section{Introdução}

A violência pode causar problemas mentais? Existiriam tipos de violência mais propícios a essa relação ou existiriam pessoas mais propensas a desenvolver problemas mentais, caso sejam vítimas de alguma violência? São perguntas que são feitas na prática da saúde mental. Em seguida, pergunta-se como a violência age no corpo e no psiquismo das pessoas, e como se pode agir terapeuticamente nesses indivíduos.

$$
\text { Essa preocupação surge na }
$$

medida em que a violência se torna endêmica em nosso país. Apesar do avanço nos índices de melhora da saúde e da pobreza, houve aumento nos índices de criminalidade (homicídios, roubos, sequestros etc.). Entre 1980 e 2000, mais de 2 milhões de pessoas morreram em virtude da violência, com aumento da ordem de $130 \%{ }^{(1)}$. Dessas, 600.000 foram homicídios, sendo dois terços na segunda década, mostrando a tendência para o crescimento.

A taxa de mortalidade por homicídio, por 100.000 habitantes, no período de 1998-2002, na cidade de Diadema, foi de 122,2, sendo a maior taxa do Brasil. São Paulo é a 25 a cidade nesse ranking, com 66,7 homicídios/100.000 habitantes, comparando-se com a cidade de Nova Iorque que teve taxa de homicídio de 6,64/100.000 habitantes, no mesmo período. Esses dados mostram a triste realidade: a violência letal, e o homicídio é fenômeno nacional.

Há preparo para lidar com esse aumento da violência? O organismo do ser humano desenvolveu, durante milhões de anos de, evolução, sofisticado mecanismo de alarme, fundamental à 
sobrevivência. A natureza nos equipou com habilidades defensivas específicas para manter o bem-estar e a segurança. Não há mais os predadores naturais no cotidiano, mas esse sistema funciona com comportamentos a ele ligado, aparecendo em situações de emergência.

Assim que algo se apresenta ameaçador, há uma reação. Essa reação inicialmente é orgânica, no sistema cardiovascular, hormonal, permitindo uma reação imediata, para, em seguida, apresentar a reação psicológica. Inicialmente, há descarga de adrenalina, via sistema simpático, e de cortisol, via sistema hipotálamo-pituitária-adrenal. Qualquer indivíduo saudável, ao passar por situação de estresse intenso, com risco de sua integridade ou de alguém próximo a ele, desenvolve, assim, reação aguda ao estresse, caracterizada por choro, raiva, medo ou insônia, todas respostas normais, esperadas. Em cerca de 30 dias esses comportamentos vão cedendo, sendo que a maioria das pessoas deixa de apresentar esses sintomas de forma mais intensa, passado esse período. A vivência traumática vai sendo elaborada e ficando apenas uma memória, também chamada declarativa, que se pode contar a respeito. É um processo fisiológico esperado.

Alguns indivíduos, mesmo nesse período inicial, podem apresentar sintomas mais intensos e disfuncionais, apresentando sintomas dissociativos, com alterações qualitativas da consciência, muitas vezes não sabendo onde está ou até quem se é (alterações da consciência auto e alopsíquica), com queixas como se sentir distante, frio, ainda com imagens repetidas do trauma vindo à sua mente, contrárias à sua vontade. Apresentam medo de serem novamente vitimizados, 
se esquivando de várias situações, devido ao mesmo. Se esses sintomas se apresentam até 30 dias do evento traumático, esse indivíduo recebe o diagnóstico de transtorno de estresse agudo (TEA).

A conduta intuitiva, realizada por muitos profissionais, no atendimento de vítimas de violência é de medicar com ansiolíticos e fazer com que o paciente fale sobre o ocorrido. Porém, os estudos têm mostrado que ambos são prejudiciais, futuramente, aos pacientes, aumentando as chances de desenvolver transtorno de estresse pós-traumático ${ }^{(2-6)}$.

Esses achados indicam reforço ao primeiro mandamento da medicina, primum non nocere, antes de tudo não prejudicar. O profissional deve avaliar primeiramente se o indivíduo está apresentando sintomas de TEA, ou se está em fase de elaboração normal da vivência traumática. Não se deve nem psicologizar ou medicalizar um conflito, quando esse não se apresenta de forma patológica. Nesses casos, deve ser providenciado suporte, conforto, orientação e educação. Nos casos de TEA, deve-se recomendar tratamento medicamentoso com inibidores seletivos de recaptação de serotonina, às vezes associado a antipsicóticos e terapia de suporte, tanto para o paciente como para os familiares.

Alguns indivíduos podem apresentar sintomas após 30 dias, algumas vezes até 1 ano da data do evento traumático, desde que esse tenha levado ao risco de vida ou à integridade física do paciente ou de alguém próximo. O paciente receberá o diagnóstico de transtorno de estresse pós-traumático (TEPT), caso apresente sintomas classificados em 3 grupos: revivência, evitação e hipervigilância. 
Os sintomas de revivência se caracterizam por pensamentos recorrentes e intrusivos, que causam sofrimento, com conteúdo relacionado ao evento (imagens, pensamentos ou percepções). Esses podem também se apresentar sob a forma de sonhos vívidos. Algumas vezes o indivíduo pode passar a agir ou sentir como se o evento estivesse acontecendo novamente, na realidade, ele sente como se estivesse de volta à cena, também chamado flashback. Todas essas revivências causam intenso sofrimento psicológico, devido à exposição aos sinais internos ou externos que simbolizam ou se assemelhem aos evento.

Devido a esse sofrimento, os pacientes fazem esforços para evitar os pensamentos, sentimentos, ou conversas associadas ao evento traumático, assim como para evitar atividades, locais ou indivíduos que tragam recordações. São os sintomas pertencentes ao agrupamento de evitação e anestesiamento afetivo. Dentro desse grupo de sintomas, aparecem os sintomas dissociativos, quando o paciente se queixa da incapacidade de relembrar um aspecto importante do trauma, ou todo o trauma e apresenta diminuição marcada do interesse ou da atividade, assim como restrição do afeto e incapacidade para experimentar o prazer (niilismo).

Por fim, o paciente apresenta também sintomas de hipervigilância, caracterizados pela dificuldade para pegar no sono, irritabilidade e crises de raiva, dificuldade de concentração e sobressaltos.

O TEPT é quadro prevalente, principalmente em locais onde existe maior violência como é o caso do Brasil. Estudos de Ribeiro e Andreoli mostraram números de prevalência, em 1 ano, ao 
redor de 4-5\%, nas cidades de São Paulo e Rio de Janeiro $^{(7)}$. Além disso, a violência provoca grande impacto no indivíduo e na sociedade, também, por sua morbidade. Os sintomas são crônicos, raramente têm remissão sem tratamento, levando muitas vezes à incapacitação do paciente acometido ${ }^{(8-9)}$.

Existem profundas alterações no funcionamento do organismo nesses pacientes, demonstrando o nível de gravidade do problema ${ }^{(10)}$.

Com o intuito de estudar e atender vitimas de violência, em São Paulo, foi criado o Programa de Atendimento e Pesquisa das Vítimas da Violência (Prove), dentro do Departamento de Psiquiatria da Universidade Federal de São Paulo (Unifesp), em fins de 2004. O mesmo teve crescimento nesse período, tornando-se referência no estudo da violência e saúde mental.
O Prove, atualmente, reformulou sua triagem e primeiro atendimento aos pacientes, criando, separadamente, dias para crianças e adolescentes e para adultos. A triagem é multidisciplinar e é realizada por miniequipes, compostas por 2 médicos, 2 psicólogas, 1 assistente social e 1 enfermeiro, todos com especialização em saúde mental.

O paciente, ao entrar no programa, é acolhido pelo profissional de enfermagem, que faz a pré-triagem, se a demanda é do Prove, e sobre a gravidade. Esse acolhimento tem o intuito de dar suporte, fundamental, para iniciar a quebra de medos e resistências, potencializadas em indivíduos vitimizados, que, naturalmente, passam desconfiar do outro. Concomitantemente, os pacientes passam pela consulta psiquiátrica, onde são avaliados se apresentam transtornos ou não, e, 
dependendo do diagnóstico, serão encaminhados para diferentes grupos dentro do Prove. Na sequência, são avaliados por profissionais da psicologia e serviço social, todos no mesmo período, com discussão da equipe para planejamento terapêutico do paciente.

Paralelamente ao ambulatório no Hospital São Paulo, é realizado, no Prove, atendimento em campo avançado, em Capão Redondo, bairro carente, localizado na periferia de São Paulo, região bastante violenta.

Entre maio e novembro de 2009,

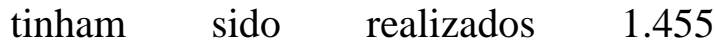
atendimentos, no Prove, média de 180 atendimentos por mês.

\section{Material e Métodos}

Foi realizado levantamento de todos os casos que deram entrada no programa, no período de maio a novembro de 2009. O levantamento foi realizado em livro de registro de atendimentos de triagem. Esse período representa a data de início de um novo esquema de triagem no Prove, do ambulatório do Hospital São Paulo. Todos os casos anotados nesse levantamento tiveram seus prontuários retirados para análise. Foi criada uma ficha com dados sociodemográficos e clínicos, a partir dos prontuários. Os dados foram coletados, tabulados e analisados.

Os dados sociodemográficos pesquisados foram: gênero, idade, estado civil, escolaridade e religião. Os dados clínicos foram colhidos através da 
anotação do diagnóstico psiquiátrico no prontuário do paciente, que foi dado após a realização de entrevista diagnóstica semiestruturada (SCID-I), por um profissional treinado. Todos os pacientes do ambulatório são submetidos a uma bateria de entrevistas e escalas psicométricas na sua admissão. Os eventos traumáticos são anotados durante essa aplicação. Alguns dados sobre a evolução do paciente, assim como o tipo de tratamento, ao qual o mesmo foi submetido, eram preenchidos na ficha com dados do prontuário.

\section{Resultados}

Foram atendidos 76 novos pacientes, sendo $52(68,4 \%)$ mulheres. A maioria (50-65\%) apresentava diagnóstico de TEPT, 23 (30,2\%) transtorno depressivo maior, 14 (18,4\%) transtorno do pânico, 8 (10,5\%) transtorno borderline de personalidade e 13 (17\%) com outros diagnósticos. Muitos casos tinham mais de um diagnóstico.

Entre esses, $44 \quad(57,8 \%)$ permaneciam em atendimento, e somente 10 (13,1\%), em 6 meses, haviam abandonado, 8 (10,5\%) haviam recebido alta, 36 (47\%) foram encaminhados para psicoterapia no próprio Prove e 14 $(18,4 \%)$ foram encaminhados para outros serviços.

As causas que levaram os pacientes adultos ao Prove estão descritas na Tabela 1. 
Tabela 1 - Frequência de eventos traumáticos motivadores da entrada no Prove

\begin{tabular}{lcc}
$\begin{array}{l}\text { Eventos traumáticos/motivo do } \\
\text { encaminhamento n=76 }\end{array}$ & $\mathbf{N}^{\mathbf{0}}$ & $\mathbf{\%}$ \\
\hline Assalto & 21 & 28 \\
Sequestro & 10 & 13 \\
Assassinato na família & 9 & 12 \\
Morte na família & 7 & 9,2 \\
Abuso sexual & 8 & 8,5 \\
Violência doméstica & 5 & 6,5 \\
Acidente com meio de transporte & 5 & 6,5 \\
Presenciar assassinato & 3 & 4 \\
Agressão física & 3 & 4 \\
Rebelião & 2 & 3 \\
Outros & 3 & 4 \\
\hline
\end{tabular}

Foram incluídas, também, 27 crianças no

Prove, nesse período, sendo 14 (52\%) meninos e 13 (48\%) meninas. Dessas, 17 (63\%) tinham entre 0 e 10 anos de idade e os demais entre 11 e 17 anos. As causas do encaminhamento entre crianças estão descritas na Tabela 2.

\section{Discussão}

A violência pode provocar problemas de saúde mental em suas vítimas. O transtorno de estresse agudo e transtorno de estresse pós-traumático são duas condições específicas, causadas pela violência. São quadros graves, com repercussões biológicas, psicológicas e sociais profundas. Devido à violência endêmica em nosso pais, e a alta prevalência dos quadros referidos, esse tema tem relevância de problema de saúde pública, devendo merecer atenção dos profissionais de saúde e autoridades. 
Tabela 2 - Causas de encaminhamento entre crianças

\begin{tabular}{lcc}
\hline \multicolumn{1}{c}{$\begin{array}{c}\text { Eventos traumáticos/motivo do } \\
\text { encaminhamento }\end{array}$} & $\begin{array}{c}\mathbf{N}^{\mathbf{0}} \\
\text { pessoas }\end{array}$ & \% \\
\hline Assassinato de familiar & 6 & 22,2 \\
Abuso sexual (inclui suspeita) & 5 & 18,5 \\
Assalto (vivido ou presenciado) & 4 & 14,81 \\
Violência doméstica (vivida ou presenciada) & 4 & 14,81 \\
Acidente (atropelamento, queda avião) & 2 & 7,4 \\
Sequestro & 2 & 7,4 \\
Mortes por doença na família & 2 & 7,4 \\
Suicídio na família & 1 & 3,7 \\
Filicídio & 1 & 3,7 \\
Negligência & 1 & 3,7 \\
Outros & 1 & 3,7 \\
\hline
\end{tabular}

\section{Refêrencias}

1. IBGE. Pesquisa Síntese dos Indicadores Sociais. 2006. Disponível em:

http://www.ibge.gov.br/home/estatistica/p opulacao/condicaodevida/indicadoresmini $\underline{\text { mos/sinteseindicsociais2006/default.shtm }}$

2. Asnis GM, Kohn SR, Henderson M, Brown NL. SSRIs versus non-SSRIs in post-traumatic stress disorder: an update with recommendations.

Drugs.

2004;64(4):383-404.

3. Davidson JR, Stein DJ, Shalev AY, Yehuda R. Posttraumatic stress disorder: acquisition, recognition, course, and treatment. J Neuropsychiatr Clin Neurosci. 2004 Spring;16(2):135-47.

4. Gelpin E, Bonne O, Peri T, Brandes D, Shalev AY. Treatment of recent trauma survivors with benzodiazepines: a prospective study. J 
Clin Psychiatry. 1996 Sep;57(9):390-4.

5. Mellman TA, Bustamante V, David D, Fins AI. Hypnotic medication in the aftermath of trauma. J Clin Psychiatry. 2002 Dec;63(12):1183-4.

6. Mayou RA, Ehlers A, Hobbs

M. Psychological debriefing for road traffic accident victims. Three-year follow-up of a randomised controlled trial. Br J Psychiatry. 2000 Jun;176:58993.

7. Andreoli S, Ribeiro, WS, Quintana, MIS, Mari, JJ, editor. Epidemiologia do transtorno de estresse pós-traumático na cidade de São Paulo: prevalência, comorbidades e fatores associados. $1^{\circ}$ Simpósio Internacional de Violência e Saúde Mental; 2008; São Paulo: UNIFESP; 2008.
8. Kilpatrick DG, Acierno R. Mental health needs of crime victims: epidemiology and outcomes. J Trauma Stress. 2003 Apr;16(2):119-32.

9. Kluznik JC, Speed N, Van Valkenburg C, Magraw R. Forty-year follow-up of United States prisoners of war. Am J Psychiatry. 1986 Nov;143(11):1443-6.

10. Ruiz J, Barbosa J Neto, Schoedl AF, Mello MF.

Psychoneuroendocrinology of posttraumatic stress disorder. Rev Bras Psiquiatr. 2007;29(supl.1):7-12.

11. Spitzer RL, Williams JB, Gibbon M, First MB. The Structured Clinical Interview for DSM-III-R (SCID). I: History, rationale, and description. Arch Gen Psychiatry. 1992;49:624-9. 


\section{Como citar este artigo:}

Serafim PM, Mello PM. Transtornos de estresse agudo e pós-traumático. SMAD, Rev. Eletrônica Saúde Mental Álcool Drog. (Ed. port.) [Internet]. 2010 [acesso: dia mês abreviado com ponto ano]; 6(Especial):460-70. Disponível em: Endereço Eletrônico Visitado. 